\title{
NGC 1817: the richest population of $\delta$ Scuti stars
}

\author{
M. F. Andersen ${ }^{1}$, T. Arentoft ${ }^{1}$, S. Frandsen ${ }^{1}$, \\ L. Glowienka ${ }^{1}$, H. R. Jensen ${ }^{1}$, and F. Grundahl ${ }^{1}$ \\ ${ }^{1}$ Department of Physics and Astronomy, Aarhus University, \\ Ny Munkegade 120, Bldg. 1520, 8000 Århus C., Denmark
}

\begin{abstract}
We combined two sets of data to get high-precision time-series for stars in the open cluster NGC 1817. We verified most of the variable stars detected earlier and found some new ones. To the $12 \delta$ Scuti variables detected prior to this article 4 new $\delta$ Scuti stars and one candidate have been added. In addition 3 $\gamma$ Doradus candidates have been detected. The total number of variables and possible variables in NGC 1817, or in the field of view of the cluster is now up to 26 . This high number of pulsating stars and especially $\delta$ Scuti variables makes this cluster an interesting asteroseismic target for future study. Using asteroseismology on $\delta$ Scuti stars will hopefully provide us with information that eventually will constrain the theoretical models of stars. This will in the end give us a much greater understanding of the evolution of stellar objects.
\end{abstract}

Accepted: 2009, July 8

Individual Objects: NGC 1817

\section{Introduction}

Asteroseismic study of pulsating stars is a very interesting field in astronomy nowadays. Results from asteroseismic investigation of oscillation modes of variable stars are helping us to understand fundamental parameters of stars. $\delta$ Scuti variable stars are stars in the range from $A$ to $F$ types and with masses ranging from $1.5 \mathrm{M}_{\odot}$ to $2.5 \mathrm{M}_{\odot}$. These stars are main sequence or post-main sequence stars and lie at the bottom of the classical Cephei instability strip. $\delta$ Scuti stars show multiperiodic signals with periods of the order of hours and magnitude variation at the mmag level. Through asteroseismology it is possible to constrain the stellar models, and to test the stellar physics to higher accuracy. 
In the open cluster NGC 1817 different kinds of variable stars are present. A high number of $\delta$ Scuti stars and at least 2 eclipsing binaries make this cluster a prominent target for asteroseismic investigation. Observations of the area including NGC 1817 made detection of at least $16 \delta$ Scuti variables possible. 12 of these were detected prior to this article (Frandsen \& Arentoft 1998 and Arentoft et al. 2005) but the last 4 are new detections. Another 4 stars are possible candidates. Open clusters are interesting targets for CCD photometry, because of the semicrowded field, which make precise time-series photometry for the individual stars possible. For NGC 1817 the turnoff from the main sequence, due to the exhaustion of Hydrogen in the core of the stars, is located inside the instability strip. This makes NGC 1817 an obvious choice for CCD observations, because the stars in the instability strip, the pulsating candidates, are among the brightest stars in the cluster.

\section{Observations}

Two datasets were used for the analysis of variable stars in the open cluster NGC 1817. The first set of data was taken with the $1.5 \mathrm{~m}$ Danish Telescope at La Silla, Chile. The observations were carried out by Lars Glowienka and Henrik Robenhagen Jensen in January 2005. The DFOSC instrument was used to obtain time-series CCD images. A $2048 \times 2048$ CCD camera was used for the I, $B$ and $V$ filter data. The main part of the total observations were carried out using the $B$ filter and a small part with the $V$ and I filter. In Fig. 1 an image from the La Silla data is shown with the detected variables indicated.

The second dataset was collected using the $2.56 \mathrm{~m}$ Nordic Optical Telescope (NOT) at La Palma, Canary Islands. These observations were performed in January 2007 by Lars Glowienka. A mosaic of four 1024 × 1024 CCD cameras were used, and to cover even more, three areas of the cluster were observed. Two images were taken in one area, then the telescope was pointed to a new area where two images were taken, and then pointed to the last area where two images were taken. Then it was moved back to the first area again and so on. One night (13-01-2007) the observations were done with the $\mathrm{V}$ filter. The last 3 nights $(14,15,16-01-2007)$ were observed with the B filter.

\subsection{Data reduction}

The two datasets were reduced separately using the Multi-Object Multi-Frame package (MOMF; Kjeldsen \& Frandsen 1992). The package uses a pointspread function (PSF) and aperture photometry to determine the magnitude of every star in the observed field. In the La Silla data, time-series for 627 stars were obtained. The reduction of the data from NOT was a little more difficult. Because of drift in the position from night to night, every CCD area was reduced separately, which resulted in 12 different MOMF-reductions per 


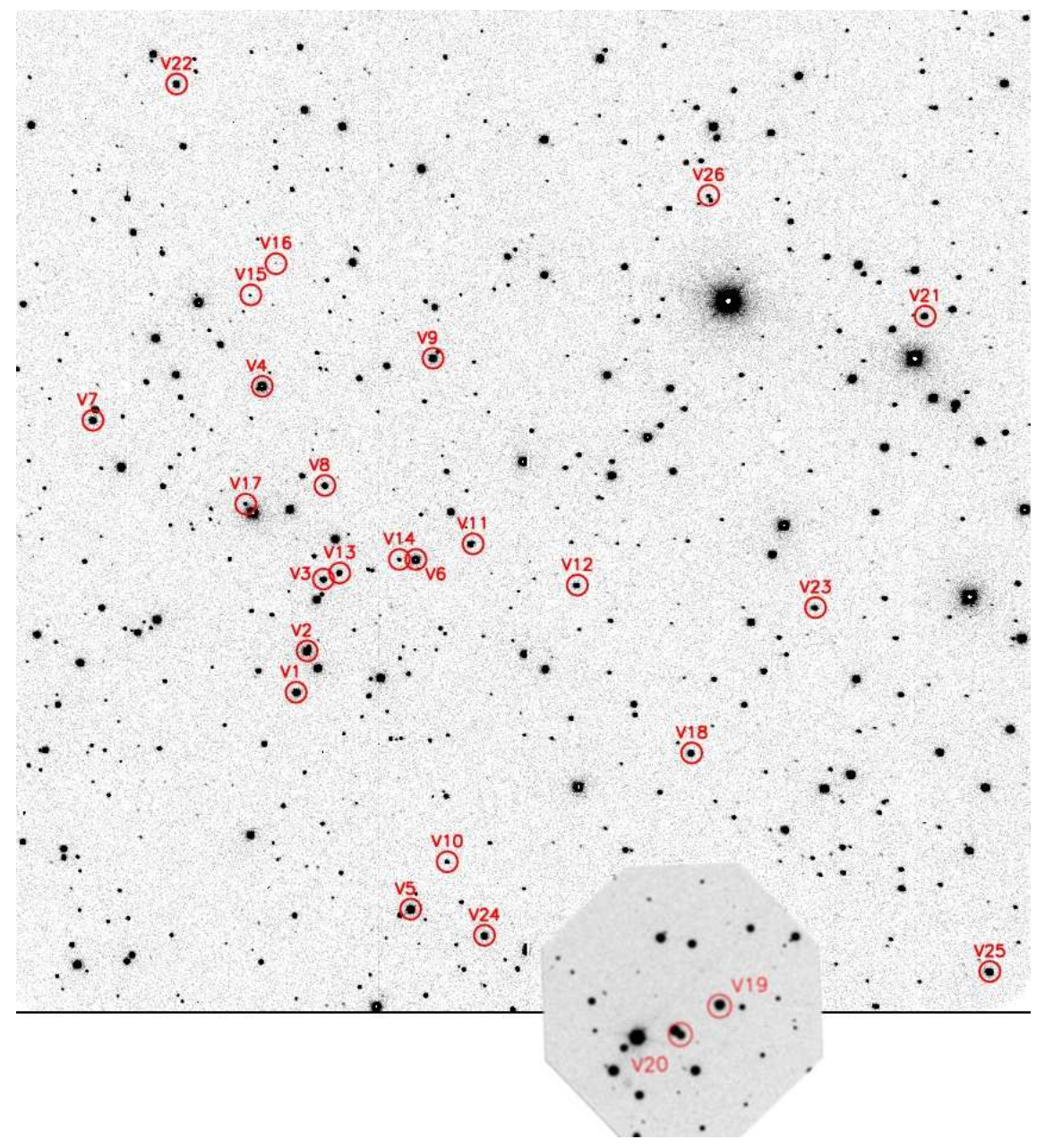

Figure 1: Finding chart for the variable stars (indicated) in NGC 1817. The La Silla field of view is $\sim 15^{\prime} \times 15^{\prime}$ and the inserted bottom image is part of one CCD image from NOT. It is rotated and scaled to match the La Silla image.

night. After the reduction, data for the detected variables were combined by normalizing the magnitude for each night. This affects the analysis of the possible $\gamma$ Doradus stars, because of their long variation periods. In the NOT data around 60 stars were present in all of the 12 areas. Time-series of around 650 stars were obtained from the NOT data, because some of the areas were overlapping. Using MOMF, a precision of $\sim 2$ mmag for the brightest stars was obtained for the La Silla data, and $\sim 1$ mmag for the NOT data. 


\section{Results}

\subsection{Detecting the variables}

The analysis of the time-series was done manually. Every star was checked visually for variability and the interesting stars were analyzed with the program Period04 (Lenz \& Breger 2005). The investigation was done with the B filter data.

In Fig. 2, a part of the light curves for the new variable stars in NGC 1817 and some candidate variables in the field is shown. The frequency solution for the stars is superimposed with a solid line. These frequencies were determined with Period04, which applies a least square fit of sinusoidals to the data. A criteria of a signal-to-noise ratio $(\mathrm{S} / \mathrm{N})$ of 4 for the detected frequencies was used (Breger et al. 1993 \& Kuschnig et al. 1997). Because of the windowfunction this criteria is not sufficient, but gives a rough estimate of the range and number of oscillations present in the stars. Long period variations were subtracted from the data when it was clear that the variations were not intrinsic. In Table 1 basic data for all the variable stars are shown, including candidates. The first 18 variables were detected by Frandsen \& Arentoft (1998) and Arentoft et al. (2005).

The $\mathrm{V}$ and $\mathrm{B}-\mathrm{V}$ magnitudes for the variable $\mathrm{V} 20$ in Table 1 were determined by comparison of 2 stars in the overlapping region for the La Silla data and the NOT data. Then the transformation was applied to V20, which was done to get an idea about the position of the star in the color-magnitude diagram (CMD). The transformation will be explained in more detail below.

\subsection{Frequency analysis}

In Table 2 the frequency solution for the new variables is listed. Only the stars for which a significant solution could be determined are shown. Oscillations with a $\mathrm{S} / \mathrm{N}$ higher than 4 were used. Because of the low number of detected frequencies, the $\mathrm{S} / \mathrm{N}$ of every solution is listed as well. The noise level was determined after removing the signals from the table.

In Fig. 2 the light curves for the new variables are shown. The solid line is the least square fit solution, with the frequencies from Table 2. Because of the reduction technique no frequency analysis was possible for the two $\gamma$ Doradus variables V23 and V24. The vertical dashed lines in these two plots are to indicate that it is not possible to combine the light curve from the different nights. Therefore no frequency analysis of these stars is possible. V21 is clearly a multiperiodic $\delta$ Scuti star (cf. Fig. 2), but the data was not sufficient for a frequency solution. 
Table 1: Basic data for the variable stars in NGC 1817

\begin{tabular}{lcccccc}
\hline ID & WIYN-ID & R.A. $(2000.0)$ & Decl. $(2000.0)$ & B & B-V & Class \\
\hline V1 & 549 & 51242.8 & 164143 & 13.50 & 0.36 & $\delta$-Scuti \\
V2 & 641 & 51240.8 & 164200 & 12.85 & 0.41 & $\delta$-Scuti \\
V3 & 788 & 51237.4 & 164231 & 14.35 & 0.45 & $\delta$-Scuti \\
V4 & 985 & 51232.2 & 164452 & 12.58 & 0.41 & $\delta$-Scuti / Binary \\
V5 & 386 & 51246.8 & 163840 & 12.86 & 0.42 & $\delta$-Scuti \\
V6 & 963 & 51233.0 & 164150 & 12.90 & 0.44 & $\delta$-Scuti \\
V7 & 650 & 51240.1 & 164607 & 13.71 & 0.39 & $\delta$-Scuti \\
V8 & 939 & 51233.7 & 164322 & 14.34 & 0.47 & $\delta$-Scuti \\
V9 & 1331 & 51224.6 & 164332 & 13.18 & 0.49 & $\delta$-Scuti \\
V10 & 534 & 51243.6 & 163846 & 16.28 & 0.46 & $\delta$-Scuti \\
V11 & 1090 & 51230.3 & 164128 & 14.28 & 0.44 & $\delta$-Scuti \\
V12 & 1203 & 51227.8 & 164007 & 14.64 & 0.50 & $\delta$-Scuti \\
V13 & 823 & 51236.5 & 164225 & 14.68 & 0.49 & $\ldots$ \\
V14 & 943 & 51233.7 & 164200 & 16.40 & 0.87 & $\ldots$ \\
V15 & 1116 & 51229.1 & 164549 & 18.23 & 1.00 & $\ldots$ \\
V16 & - & 51226.9 & 164552 & 18.91 & 1.13 & Binary \\
V17 & 773 & 51237.4 & 164356 & 16.63 & 0.72 & $\delta$-Scuti \\
V18 & 1128 & 51229.9 & 163730 & 14.17 & 0.47 & Binary \\
V19 & - & 51238.4 & 163454 & 13.28 & 0.33 & $\delta$-Scuti \\
V20 & - & 51241.2 & 163458 & $\sim 14.24$ & $\sim 0.57$ & $\delta$-Scuti \\
V21 & - & 51204.0 & 163921 & 14.37 & 0.42 & $\delta$-Scuti \\
V22 & - & 51223.8 & 164827 & 13.97 & 0.47 & $\delta$-Scuti \\
V23 & - & 51219.5 & 163741 & 14.64 & 0.46 & $\gamma$-Dor \\
V24 & 476 & 51245.0 & 163744 & 13.41 & 0.80 & $\gamma$-Dor \\
V25 & - & 51226.9 & 163242 & 13.28 & 0.67 & $\gamma$-Dor \\
V26 & - & 51207.6 & 164229 & 16.81 & 0.69 & $\delta$-Scuti \\
\hline
\end{tabular}

The variable ID for the first 18 stars is that given by Arentoft et al. (2005) and the WIYN-ID is the star number from the WIYN catalog. Right ascension is given in hours, minutes and seconds and declination is given in degrees, arcminutes and arcseconds.

Table 2: Pulsational data for the new variables in NGC 1817

\begin{tabular}{cccccccc}
\hline ID & $f_{1}$ & $(S / N)_{1}$ & $f_{2}$ & $(S / N)_{2}$ & $f_{3}$ & $(S / N)_{3}$ & Class \\
\hline V19 & 10.89 & 25.7 & 11.73 & 21.7 & 14.83 & 4.57 & $\delta$-Scuti \\
V20 & 24.05 & 5.21 & $\ldots$ & $\ldots$ & $\ldots$ & $\ldots$ & $\delta$-Scuti \\
V22 & 14.15 & 9.14 & $\ldots$ & $\ldots$ & $\ldots$ & $\ldots$ & $\delta$-Scuti \\
V25 & 0.3507 & 130 & $\ldots$ & $\ldots$ & $\ldots$ & $\ldots$ & $\gamma$-Dor \\
V26 & 4.653 & 5.80 & 6.112 & 4.90 & $\ldots$ & $\ldots$ & $\delta$-Scuti \\
\hline
\end{tabular}

The frequencies are given in cycles per day and the $\mathrm{S} / \mathrm{N}$ ratio is shown for every frequency.

\subsection{The color-magnitude diagram}

A transformation from the observed $V$ and $B$ data, from La Silla, was done using the WIYN Open Cluster Study (Wisconsin, Indiana, Yale and NOAO) catalog of standardized CCD photometry. 96 stars, which were identified in both WIYN and the La Silla data, were used to obtain the offset transformation. The 


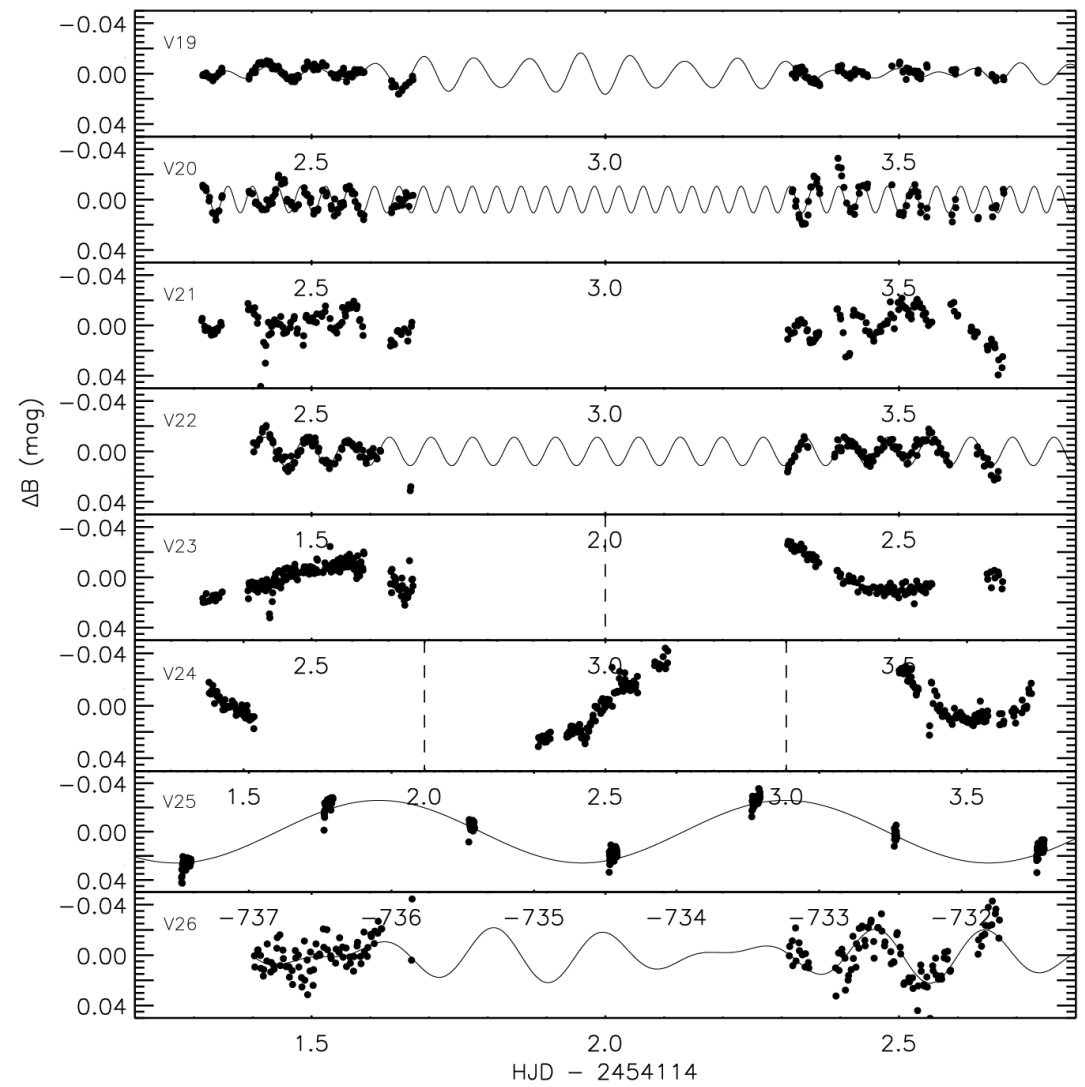

Figure 2: Light curves for the 8 new variables

position of the stars was used to match the different stars in the two data sets.

In Fig. 3 the CMD for the stars in the observed field from La Silla is shown. The variable stars are indicated; diamonds indicate $\delta$ Scuti variables, squares indicate binary systems, triangles indicate $\gamma$ Doradus variables and circles indicate candidate variables.

The instability strip (Breger 2000) for NGC 1817 is superimposed with the dashed lines. It is evident that most of the variables are located inside the instability strip. We also see that the stars inside the strip are positioned close to the cluster sequence, which means that most of the stars in the instability strip are likely cluster members. Errors in the CMD are dominated by transformation errors, but the figure is in good agreement with the CMD determined 


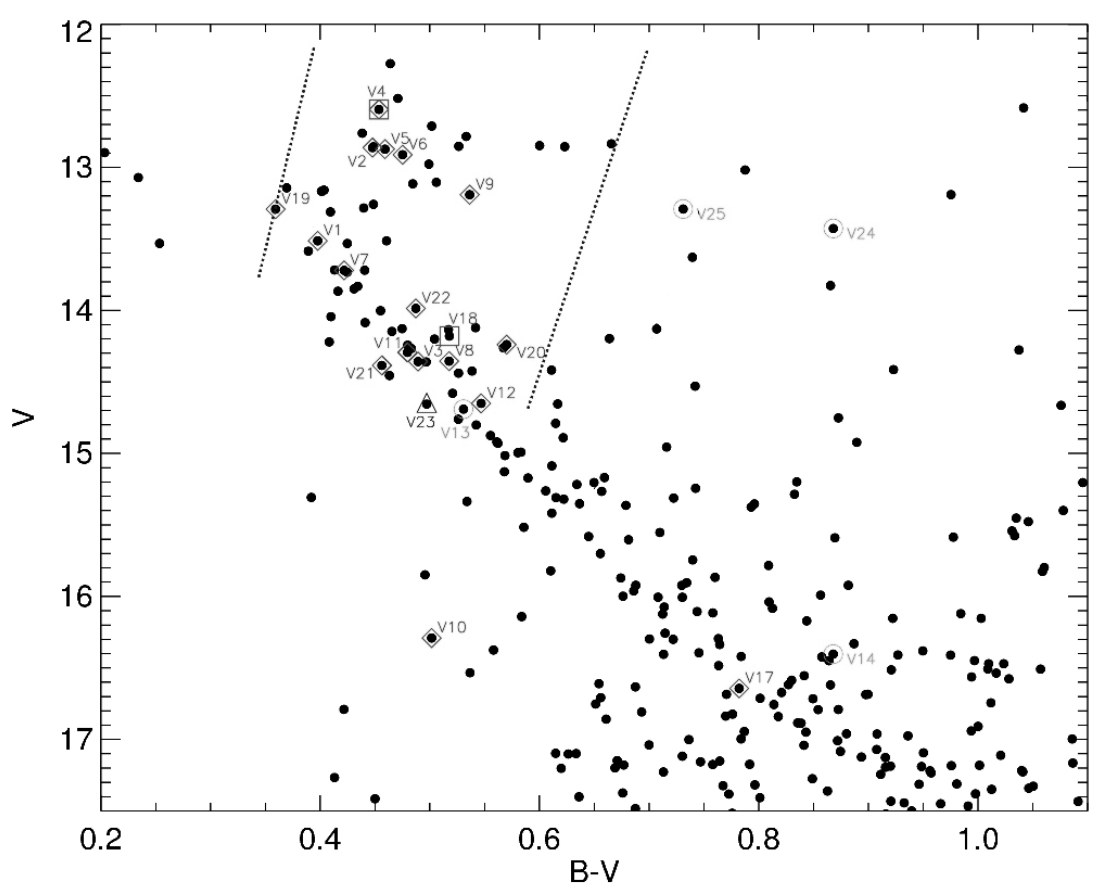

Figure 3: Color-Magnitude diagram for stars in i NGC 1817

by Arentoft et al. (2005).

\section{Conclusion}

The detection of $17 \delta$ Scuti variables, 2 eclipsing binaries (one with a component that is also a $\delta$ Scuti) and 8 other possible variables, 4 which could be $\gamma$ Doradus variables, one contact binary, one $\delta$ Scuti and 2 other candidates, is at the moment the highest concentration of $\delta$ Scuti variables in any open cluster. This makes NGC 1817 a very interesting asteroseismic target. The combination of $\delta$ Scuti stars and eclipsing binary systems in an open cluster makes this particular target of greatest interest.

Membership determination of the variables by considering the proper motion, and spectroscopic study of the eclipsing binaries will be the next step. J. Molenda-Żakowitz et al. (2009) used spectroscopic observations of 11 variable stars in NGC 1817 to analyze these stars further. In the future a multisite campaign will be needed to get sufficient time-series data to make asteroseismic 
study of the individual stars possible.

\section{References}

Arentoft, T., Bouzid, M. Y., Sterken, C. et al. 2005, PASP, 117, 601

Balaguer-Núñez, L., Jordi, C., Galadí-Enríquez, D., et al. 2008, A\&A, 426, 819

Breger, M., Stich, J., Garrido, R. et al. 1993, A\&A, 271, 482

Breger, M. 2000, ASPC, 210, Proceedings of Delta Scuti and Related Stars, ed. M. Breger \& M. H. Montgomery (San Francisco: ASP), 3

Frandsen, S., \& Arentoft, T. 1998, A\&A, 333, 524

Kjeldsen, H., \& Frandsen, S. 1992, PASP, 104, 413

Kuschnig, R., Weiss, W. W., Gruber, R., et al. 1997, A\&A, 328, 544

Lenz, P., \& Breger, M. 2005, CoAst, 146, 53

Machado, L. Fox., Schuster, W. J., Zurita, C., et al. 2008, CoAst, 156, 27

Molenda-Żakowitz, J., Arentoft, T., Frandsen, S., et al. 2009, AcA, 59, 69 